\title{
Fast Testing of Critical Properties through Passive Testing ${ }^{\star}$
}

\author{
José Antonio Arnedo ${ }^{1}$, Ana Cavalli ${ }^{1}$, and Manuel Núñez ${ }^{2}$ \\ 1 Institut National des Télécommunications GET-INT \\ 91011 Evry Cedex, France \\ \{Jose-Antonio. Arnedo-Rodriguez, Ana.Cavalli\}@int-evry.fr \\ 2 Dept. Sistemas Informáticos y Programación \\ Universidad Complutense de Madrid, E-28040 Madrid, Spain \\ mn@sip.ucm.es
}

\begin{abstract}
We present a novel methodology to perform passive testing. The usual approach consists in recording the trace produced by the implementation under test and trying to find a fault by comparing this trace with the specification. We propose a more active approach to passive testing where the minimum set of (critical) properties required to a correct implementation may be explicitly indicated. In short, an invariant expresses that each time that the implementation under test performs a given sequence of input/output actions, then it must show a behavior reflected in the invariant. By using an adaptation of the classical pattern matching algorithms on strings, we obtain that the complexity of checking whether an invariant is fulfilled by the observed trace is in $\mathcal{O}(n \cdot m)$, where $n$ and $m$ are the lengths of the trace and the invariant, respectively. If the length of the invariant is much smaller than the length of the trace then this complexity is almost linear with respect to the length of the trace. Actually, this is usually the case for most practical examples. In addition to our methodology, we present the case study that was the driving force for the development of our theory: The Wireless Application Protocol (WAP). We present a test architecture for WAP as well as the experimental results obtained from the application of our passive testing with invariants approach.
\end{abstract}

\section{Introduction}

The main purpose of testing is to find out whether an implementation presents the behavior that was indicated by the corresponding specification. In order to perform this task, several techniques, algorithms, and semantic frameworks have been introduced in the literature (see e.g. [LY96,Lai02] for two overviews on the topic). Most of the proposals for testing are based on the so-called active testing. Intuitively, the tester sends an input to the implementation and waits

\footnotetext{
* Research supported in part by the Spanish Ministerio de Ciencia y Tecnología projects MASTER and AMEVA. This research was carried out while the third author was visiting the GET-INT.
} 
for an output. If the output belongs to the expected ones, according to the specification, then the process continues; otherwise, a fault has been detected in the implementation. This kind of testing is called active because the tester has total control over the inputs provided to the implementation under test. On the contrary, passive testing (see e.g. [AAD79,LNS ${ }^{+}$97,Mil98,TC99,TCI99]) does not involve the presence of an active tester. In passive testing the implementation under test is allowed to run independently without any interaction with a tester. However, the trace that the implementation is executing is observed so that it can be analyzed. By comparing the obtained trace with the specification we may detect some faults in the implementation.

Unfortunately, passive testing is less powerful than active testing. This is so because active testing allows a closer control of the implementation under test. For example, depending on the received output, we may choose among a set of inputs to be applied to the implementation. In passive testing this capability is lost. Thus, it can happen that faults that could be detected by choosing an appropriate input are not found because the implementation does not take that path. Nevertheless, passive testing presents some important advantages. First, active testing is in general more costly because the testing process has to be closely controlled. Second, there are situations where active testing is not even feasible. For instance, passive testing is attracting a lot of study in the field of network management where the testing process has to be minimized in order to reduce the use of the network (see [MA01a,MA01b,WZY01] for some recent works on the topic).

Even though there is ongoing work on passive testing ${ }^{1}$ most of these proposals share a common pattern. Usually, the trace is taken and the specification is traversed in order to detect a fault. A drawback of this approach is that it presents a low performance (in terms of complexity in the worst case) if non-deterministic specifications are considered. A notable exception is presented in [CGP01] where the specification is (partially) set apart. Actually, they extract some test sequences from the specification, called invariants, and they check whether the trace obtained from the implementation under test is correct with respect to them. So, once these test are extracted, the specification plays no role in the testing process. They consider three kinds of invariants. However, the most useful for practical purposes are the so-called output invariants. Intuitively, an (output) invariant as $i_{1} / o_{1}, \ldots, i_{n-1} / o_{n-1}, i_{n} / o_{n}$ must be interpreted as "each time the implementation performs the sequence $i_{1} / o_{1}, \ldots, i_{n-1} / o_{n-1}, i_{n}$ the next observed output must be $o_{n}$ ".

This paper extends and improves [CGP01] in several ways. Actually, our methodology represents a real example of theory guided by practice. In fact, we came out with our different notions of invariants when we tried to apply [CGP01] to a real protocol. By doing so we found some shortcomings. First, their invariants were not expressive enough for our purposes. For example, the complete sequence has to be indicated. That is, properties as

\footnotetext{
${ }^{1}$ Nevertheless, the effort spent in passive testing is not yet comparable with the one for the study of active testing techniques.
} 
Each time that a user asks for connection and the connection is granted, if after performing some operations the user asks for disconnection then he is disconnected.

cannot be easily represented by using their invariants, because all the possible sequences of actions expressing the idea of some operations must be explicitly written. So, we have added the possibility of specifying wild-card characters in invariants. Besides, we now allow a set of outputs (instead of a single output) as termination of the invariant. Thus, we may specify properties as

Each time that a user asks for a resource (e.g. a web page) either the resource is obtained or an error is produced.

Finally, their invariants were automatically extracted from the specification. This approach presents two drawbacks. First, interesting invariants cannot be distinguished from trailing invariants. Second, and more important, the complexity of extracting invariants exponentially increases with their length. That is, the complexity of extracting the invariants of length $n$ is in $\mathcal{O}\left(|\operatorname{Tr}|^{n}\right)$, where $|T r|$ is equal to the number of transitions in the specification. We claim that invariants should be supplied by the specifier/tester. In this case, the first step must be to check that the invariant is in fact correct with respect to the specification. We provide an algorithm that checks this correctness in linear time, with respect to the number of transitions, if the invariant does not contain the wild-card character $*$; this complexity is quadratic if the symbol $*$ appears in the invariant.

Once we have a set of (correct) invariants, our approach to passive testing proceeds as follows: We observe the trace produced by the implementation under test and we decide whether this trace respects the invariants. In order to do so, we have implemented a simple variant of the classical algorithms for pattern matching on strings (see e.g. [BM77,KMP77]). Our algorithm works, in the worst case, in time $\mathcal{O}(m \cdot n)$, where $n$ and $m$ are the length of the trace and the length of the invariant, respectively. Let us remark that in most practical cases the length of the invariant is several orders of magnitude smaller than the length of the trace. Thus, we may consider that the complexity is almost linear with respect to the length of the trace.

In addition to the formal framework, in this paper we also report our experiments on the WAP (Wireless Application Protocol). This protocol is an open global specification that empowers mobile users with wireless devices to easily access and interact with information and services instantly. It is worth to point out that this protocol represents a typical example where active testing cannot be applied. In general, there is no direct access to the interfaces between the different layers. Thus, the tester cannot control how internal communications are established. However, in our experiments we have used a software free protocol stack, namely Kannel, and we have the possibility of installing points of observation, in short POs, between the different layers. Moreover, a platform and a test architecture capable to deal with passive testing in a mobile phone environment (WAP, GPRS, UMTS) have been defined. The platform and the architecture are 

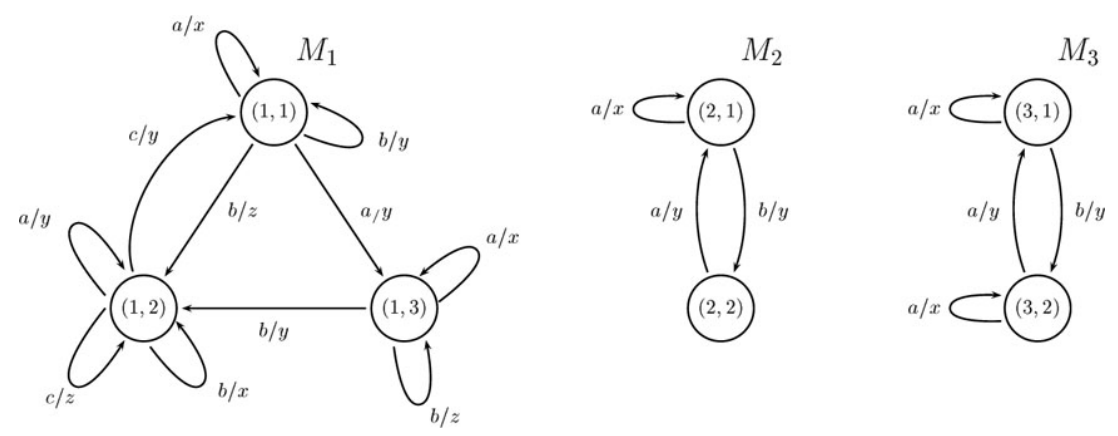

Fig. 1. Examples of FSMs.

used to apply our passive testing with invariants approach. We take the output of the platform, in the form of log files, and we apply the appropriate invariants to the obtained trace. This experiment represents an original contribution because such a study has been never performed in a systematic way.

The rest of the paper is organized as follows. In Section 2 we present our notion of invariants, that we call simple invariants. These invariants are able to express properties as "after $x$ has happened then we must have that $y$ happens". We give algorithms to decide the correctness of our invariants with respect to a given specification and we explain how our invariants are applied to the observed trace. In Section 3 we present the WAP and we briefly comment on the performed experiments by using our notion of passive testing. Finally, in Section 4 we give our conclusions and some lines for future work.

\section{Invariants and Passive Testing}

In this section we introduce our invariants and the corresponding algorithms to decide whether they are correct with respect to specifications. We consider that specifications are represented as Finite State Machines. However, we will comment on how our invariants can be extended to deal with data.

Definition 1. A Finite State Machine, in the following FSM, is a tuple $M=$ $\left(S, \mathcal{I}, \mathcal{O}, T r, s_{i n}\right)$ where $S$ is a finite set of states, $\mathcal{I}$ is the set of input actions, $\mathcal{O}$ is the set of output actions, $\operatorname{Tr}$ is the set of transitions, and $s_{\text {in }}$ is the initial state.

Each transition $t \in \operatorname{Tr}$ is a tuple $t=\left(s, s^{\prime}, i, o\right)$ where $s, s^{\prime} \in S$ are the initial and final states of the transition, respectively, and $i \in \mathcal{I}, o \in \mathcal{O}$ are the input and output actions, respectively.

Let $s, s^{\prime} \in S$ be states and $t r=i_{1} / o_{1}, \ldots, i_{n} / o_{n}$, for $n \geq 1$, be a sequence of pairs such that for any $1 \leq j \leq n$ we have $i_{j} \in \mathcal{I}$ and $o_{j} \in \mathcal{O}$. We write $s \stackrel{t r}{\longrightarrow} s^{\prime}$ if either $t r=\epsilon$ and $s=s^{\prime}$ or there exist $n$ transitions $t_{1}, \ldots, t_{n} \in \operatorname{Tr}$ and states $s_{1}, \ldots, s_{n-1} \in S$ such that $t_{1}=\left(s, s_{1}, i_{1}, o_{1}\right), t_{n}=\left(s_{n-1}, s^{\prime}, i_{n}, o_{n}\right)$, and for any $1<j<n$ we have $t_{j}=\left(s_{j-1}, s_{j}, i_{j}, o_{j}\right)$. 
First, let us note that this notion of FSM does not restrict specifications to be deterministic, so that we work with a general notion of FSM. Intuitively, a transition $t=\left(s, s^{\prime}, i, o\right)$ indicates that if the machine is in state $s$ and receives the input $i$ then the machine emits the output $o$ and the current state becomes $s^{\prime}$. We will sometimes write $s \stackrel{i / o}{\longrightarrow} s^{\prime}$ to denote that we have the transition $\left(s, s^{\prime}, i, o\right) \in \operatorname{Tr}$. We can extend the notion of single transition to a sequence of transitions. Thus, $s \stackrel{t r}{\longrightarrow} s^{\prime}$ simply denotes that we can traverse from the state $s$ to the state $s^{\prime}$ by following transitions containing the corresponding pairs $i / o$ appearing in $t r$. In Figure 1 we present some simple examples of Finite State Machines.

Once we have a FSM we may extend its set of transitions so that the wild-card characters ? and $*$ can be taken into account. In particular, these special symbols can appear as part of a sequence of transitions.

Definition 2. Let $M=\left(S, \mathcal{I}, \mathcal{O}, T r, s_{i n}\right)$ be an FSM. We write $s \stackrel{\text { ?/o }}{\longrightarrow} s^{\prime}$ (resp. $s \stackrel{i / ?}{\longrightarrow} s^{\prime}$ ) if there exists $i \in \mathcal{I}$ (resp. $o \in \mathcal{O}$ ) such that $s \stackrel{i / o}{\longrightarrow} s^{\prime}$. Besides, we write $s \stackrel{? / ?}{\longrightarrow} s^{\prime}$ if there exist $i \in \mathcal{I}$ and $o \in \mathcal{O}$ such that $s \stackrel{i / o}{\longrightarrow} s^{\prime}$. We write $s \stackrel{*}{\longrightarrow} s^{\prime}$ if there exists a sequence of input/output pairs $\operatorname{tr}$ such that $s \stackrel{t r}{\longrightarrow} s^{\prime}$.

\subsection{Introducing Simple Invariants}

Next we present our notion of invariant. We call them simple invariants, or just invariants. Let $M=\left(S, \mathcal{I}, \mathcal{O}, T r, s_{i n}\right)$ be an FSM. Intuitively, a trace as $i_{1} / o_{1}, \ldots, i_{n-1} / o_{n-1}, i_{n} / O$ is a simple invariant for $M$ if each time that the trace $i_{1} / o_{1}, \ldots, i_{n-1} / o_{n-1}$ is observed, if we obtain the input $i_{n}$ then we necessarily get an output belonging to $O$, where $O \subseteq \mathcal{O}$. In addition to sequences of input/output symbols, we will allow the wildcard characters ? and $*$. In our framework, the meaning of ? is the standard one in the pattern matching community (that is, to replace any symbol). However, we will slightly modify the usual meaning of $*$. For example, the intuitive meaning of an invariant as $i / o, *, i^{\prime} / O$ is that if we detect the transition $i / o$ then the first occurrence of the input symbol $i^{\prime}$ is followed by an output belonging to the set $O$. In other words, $*$ replaces any sequence of symbols not containing the input symbol $i^{\prime}$.

Definition 3. Let $M=\left(S, \mathcal{I}, \mathcal{O}, T r, s_{i n}\right)$ be an FSM. We say that the sequence $t r$ is a (simple) invariant for $M$ if the following two conditions hold:

1. $t r$ is defined according to the EBNF $t r::=i / O|*, t r| a / z, t r$. In the previous expression we consider $i \in \mathcal{I}, a \in \mathcal{I} \cup\{?\}, z \in \mathcal{O} \cup\{?\}$, and $O \subseteq \mathcal{O}$.

2. $t r$ is correct with respect to $M$.

We denote the set of (simple) invariants for $M$ by $\operatorname{In} v_{M}$.

In Figures 2 and 3 we introduce two algorithms to decide whether an invariant is correct with respect to a specification. First, we present some examples of invariants to show what kind of critical properties can be tested as well as how our invariants work. 
Example 1. Our notion of invariant allows us to express several interesting properties. For example, we can test that when a user requests a disconnection then he is in fact disconnected by using the invariant

$$
I_{1}=\text { req_disconnect } /\{\text { disconnected }\}
$$

The idea is that each time that the symbol req_disconnect appears in the trace then it is followed by the output symbol disconnected. For instance, this invariant has the same distinguishing power as the invariant

$$
I_{1}^{\prime}=*, \text { req_disconnect } /\{\text { disconnected }\}
$$

We can specify a more complex property by taking into account that we are interested in disconnections only if a connection was requested. In this case we have

$$
I_{2}=\text { req_connect/?,*,req_disconnect } /\{\text { disconnected }\}
$$

We can refine the previous invariant if we only consider the cases where the connection was granted

$$
I_{3}=\text { req_connect/granted_connection, } *, \text { req_disconnect } /\{\text { disconnected }\}
$$

For example, a trace is correct with respect to $I_{3}$ if each time that we find a (sub)sequence starting with the pair req_connect/granted_connection then the first occurrence of the input symbol req_disconnect is paired with the output symbol disconnected. Let us remark that it can be the case that the pair req_connect/granted_connection appears in the observed trace but that the input req_disconnect is not detected afterwards in the corresponding trace. In such a situation we cannot conclude that the implementation fails: It may happen that we have stopped too soon observing the behavior of the implementation. Finally, an invariant as

$$
I_{4}=\text { req_connect } /\{\text { granted_connection, } \text { error }\}
$$

indicates that after requesting a connection we either are granted with it or an error is produced.

We could adapt to our framework the algorithm given in [CGP01] to extract their invariants, up to a length $n$, for a specification $M$. However, as we explained in the introduction of the paper, this process presents several drawbacks. In particular, the complexity exponentially increases with the length of invariants. On the contrary, we advocate that invariants should be indicated by the specifier/tester as the set of critical properties that the implementation must fulfill. Fortunately, we have found an algorithm that detects in linear time (with respect to the number of transitions in the FSM) whether a sequence of symbols not containing the character $*$ is in fact an invariant for a specification. Obviously, before we try to find out whether the trace observed from the behavior of the implementation is correct with respect to an invariant, we should assure 
Input: $M=\left(S, \mathcal{I}, \mathcal{O}, T r, s_{i n}\right), I=i_{1} / o_{1}, \ldots, i_{n} / O, \forall 1 \leq j \leq n: i_{j} \neq * \wedge o_{j} \neq *$ Ouput: true/false $\equiv$ invariant $I$ correct/incorrect

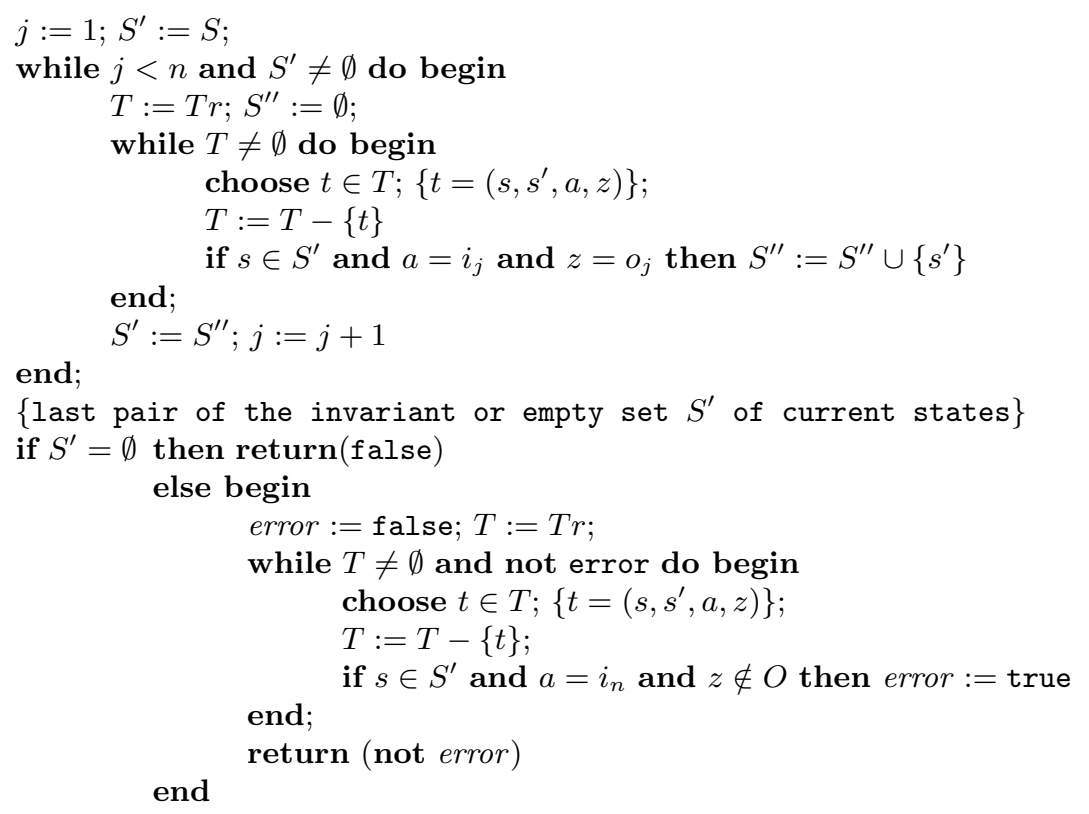

We consider that both $i=$ ? and $o=$ ? hold.

Fig. 2. Checking correctness of Invariants (1/2).

that the invariant is in fact correct with respect to the specification. In order to facilitate the reading, we first present an algorithm (see Figure 2) deciding correctness of invariants without occurrences of the $*$ wild-card character. In Figure 3 we extend this algorithm to deal with invariants where the symbol * can appear.

The algorithm given in Figure 2 works as follows. The first while-loop computes those states $s \in S$ such that they can be reached from one of the states in $S$ by following the sequence $i_{1} / o_{1}, \ldots, i_{n-1} / o_{n-1}$. Let us remark that if one of the symbols in the sequence is the wild-card character ? then any symbol can be used. Besides, it may happen that after some steps we find that there do not exist two states connected by the analyzed sub-sequence. In this case, $S^{\prime}$ becomes empty. Let us note that for each execution of the loop we perform a number of operations proportional to the number of transitions in the corresponding specification. So, in the worst case we perform a number of operations proportional to the number of transitions times the length of the sequence, that is, $n$. The second while-loop analyzes the last pair of the invariant. If the auxiliar set of states $S^{\prime}$ is empty then the invariant is incorrect. Actually, this means that there does not exist a state in the specification such that the sequence of pairs forming the invariant can be performed from it. Thus, we should not con- 
sider that this candidate represents any property of the specification ${ }^{2}$. If that set is not empty then we check that for any transition labelled by the input $i_{n}$ we receive an output belonging to $O$. Again, the complexity of this last loop is given by the number of transitions. Besides, we need $|S|+|\operatorname{Tr}|$ additional space. Let us note that if the graph induced by the corresponding FSM is connected then $|S| \leq|T r|+1$ (otherwise we can discard those states and transitions not reachable from the initial state and the result holds for the new sets of states and transitions).

Proposition 1. Let $M=\left(S, \mathcal{I}, \mathcal{O}, T r, s_{i n}\right)$ be an FSM and $I=i_{1} / o_{1}, \ldots, i_{n} / O$ be an invariant such that for any $1 \leq j \leq n$ we have $i_{j} \neq * \wedge o_{j} \neq *$. The worst case of the algorithm given in Figure 2 checks the correctness of the invariant $I$ with respect to $M$ in time $\mathcal{O}(n \cdot|\operatorname{Tr}|)$ and space $\mathcal{O}(|\operatorname{Tr}|)$.

Next, we present some examples of correct/incorrect invariants for a given specification.

Example 2. Let us consider the FSMs presented in Figure 1. For example, the following invariants are correct for $M_{1}$ :

$$
I_{1}=a /\{x, y\} \quad I_{2}=a / ?, c / z, b /\{x\}
$$

Let us remark that $I_{1}$ is also correct for both $M_{2}$ and $M_{3}$. On the contrary, $I_{2}$ is incorrect for them since the sequence $a / ?, c / z$ cannot be performed from any state belonging either to $M_{2}$ or $M_{3}$. If we consider the invariant

$$
I_{3}=b / y, a /\{y\}
$$

we have that $I_{3}$ is incorrect for $M_{1}$. For instance, we have a transition labelled by $b / y$ outgoing from the state $(1,1)$ and reaching the same state $(1,1)$. Then, we have a transition labelled by $a / x$ from the state $(1,1)$. So, there exists a state (in this case $(1,1)$ ) such that the sequence of transitions $b / y$ can be performed in such a way that the reached state (i.e. $(1,1)$ ) may perform a transition whose input action is $a$ but the corresponding output action does not belong to the set $\{y\}$. Besides, this invariant is correct for $M_{2}$ while it is incorrect for $M_{3}$.

In Figure 3 we extend the previous algorithm to deal with invariants containing the wild-card character $*$. As in the previous case, we traverse the invariant from left to right. We also have that the external while-loop has as termination condition that either the remaining sequence has length one or that the current set of states is empty. However, instead of advancing by incrementing a counter we consider two auxiliar functions: head $(I)$ returns the first element of $I$ and $\operatorname{tail}(I)$ removes the first element from $I$. If the first element of the remaining invariant is a pair $i / o$ where $i \in \mathcal{I} \cup\{?\}$ and $o \in \mathcal{O} \cup\{?\}$ then the algorithm

\footnotetext{
${ }^{2}$ Another possibility would be to consider the usual meaning of the logical implication. Thus, the predicate "each time that the prefix is performed then something happens" would hold since the premise is false.
} 
Input: $M=\left(S, \mathcal{I}, \mathcal{O}, T r, s_{i n}\right), I=i_{1} / o_{1}, \ldots, i_{n} / O$

Ouput: true/false $\equiv$ invariant $I$ correct/incorrect

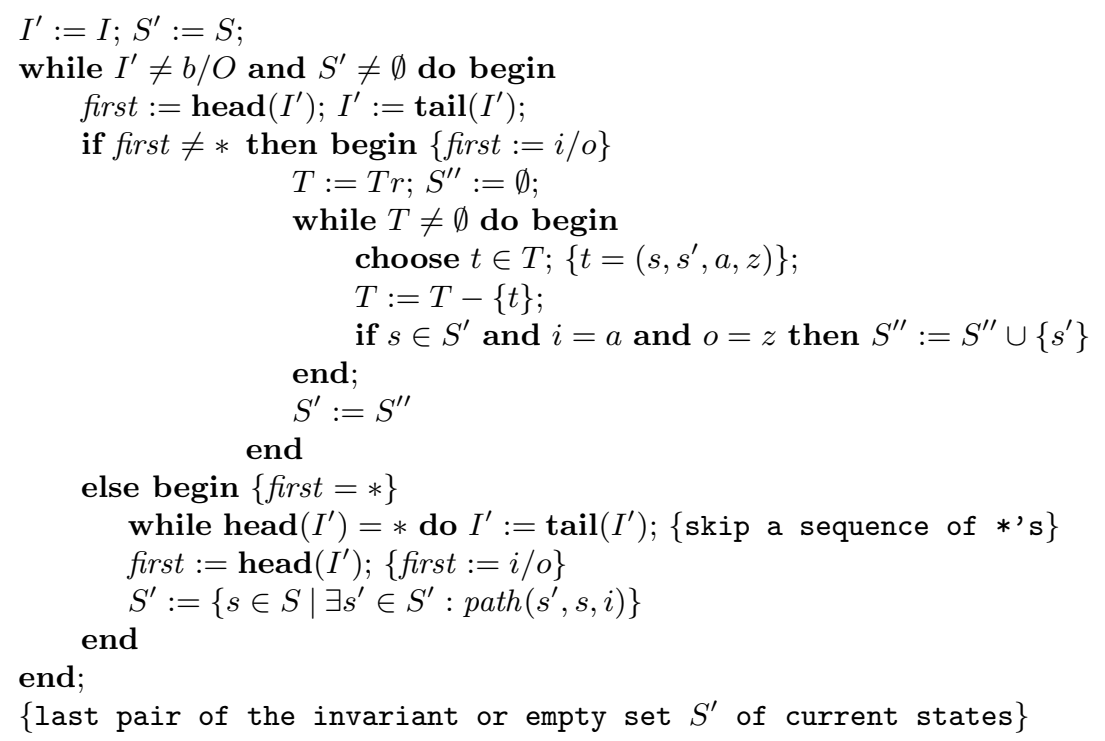

See Algorithm in Figure 2 for dealing with the last pair of the invariant.

We consider that both $i=$ ? and $o=$ ? hold.

Fig. 3. Checking correctness of Invariants $(2 / 2)$.

proceeds as the algorithm presented in Figure 2. If we have that the first element is $*$ then we skip all consecutive *'s. Afterwards, we consider the first element of the remaining trace. Let us remark that this element must be a pair $i / o$. We compute those states $s$ connected with one of the states $s^{\prime} \in S^{\prime}$ by a path that does not contain the symbol $i$. These paths are computed by the predicate path $\left(s^{\prime}, s, i\right)$. Formally, path $\left(s^{\prime}, s, i\right)$ if there exists a sequence of input/output pairs $t r=a_{1} / z_{1}, \ldots a_{r} / z_{r}$ such that $s^{\prime} \stackrel{t r}{\longrightarrow} s$ and for any $1 \leq j \leq r$ we have $a_{i} \neq i$. As a special case, if $i$ is equal to ? then $\operatorname{path}\left(s^{\prime}, s, ?\right)$ holds if $s^{\prime} \stackrel{*}{\longrightarrow} s$. Let us remark that the complexity in time in the worst case for computing this new set of states is in $\mathcal{O}(|S| \cdot|\operatorname{Tr}|)$. This is so because we only need to compute a breath-first-search (the complexity of this operation is in $\mathcal{O}(|\operatorname{Tr}|)$ ) for each of the states belonging to $S^{\prime}$ (at most $|S|$ states). Again, if we consider that the induced graph is connected then we have that the previous complexity is bounded by $\mathcal{O}\left(|T r|^{2}\right)$. Besides, we need $|S|+|T r|$ additional space. Finally, the last element of the sequence is treated as in the algorithm given in Figure 2.

The next result indicates the complexity of the previous algorithm. We consider that there are no trailing occurrences of the wild-card character $*$ in invariants, that is, no consecutive occurrences of $*$. 
Proposition 2. Let $M=\left(S, \mathcal{I}, \mathcal{O}, T r, s_{i n}\right)$ be a FSM and $I=i_{1} / o_{1}, \ldots, i_{n} / O$ be an invariant without trailing occurrences of $*$. The worst case of the algorithm given in Figure 3 checks the correctness of the invariant $I$ with respect to $M$ in time $\mathcal{O}\left(k \cdot|T r|^{2}+(n-k) \cdot|T r|\right)$, where $k$ is equal to the number of $*$ 's in $I$. Besides, the needed extra space is in $\mathcal{O}(|\operatorname{Tr}|)$.

Example 3. If we consider again the FSMs depicted in Figure 1 we have that the invariant $a / x, *, b /\{y, z\}$ is correct for all of the specifications.

Next, we have to determine whether the trace obtained from the implementation satisfies the properties indicated by the invariants that we are interested in. Let us remark a very important difference with respect to previous proposals for passive testing. That is, a homing state phase is not needed for this kind of invariants. This is so because invariants have to be fulfilled at any point of the implementation. Thus, it is not relevant the state where the machine was placed when we started to observe the trace. In order to test the trace we perform a pattern matching strategy. We have implemented a simple adaptation of the classical algorithms for pattern matching on strings (e.g. [BM77,KMP77]). The inclusion of wild-card characters is easy. In addition, for an invariant of length $n$ we have to consider all the occurrences of the first $n-1$ elements in the trace and then if we find a pair $i / o$ such that $i_{n}=i$ (let us remind that if $i_{n}=$ ? then this equality holds) then we have to check that $o \in O$. We can say that we have found a mismatch (that is, a fault) if this last condition does not hold. Regarding the complexity of our pattern matching strategy, in the worst case we obtain $\mathcal{O}(m \cdot n)$. Let us remark that even though good algorithms for pattern matching on strings perform in $\mathcal{O}(m)$ (after the pre-processing phase) we cannot achieve this complexity because we must check all the occurrences of the pattern in the trace. However, as we commented before, if we consider that the length of the invariant is much smaller than the length of the trace, as it is usually the case, we have that this complexity is almost linear with respect to the length of the trace.

We finish this section by presenting some relations between different invariants and their correctness with respect to a given specification. The proofs of these results are easy (but tedious) with respect to the algorithm given in Figure 3 .

Lemma 1. Let $M=\left(S, \mathcal{I}, \mathcal{O}, T r, s_{i n}\right)$ be an FSM. The following properties hold:

- The invariant $*, i_{1} / o_{1}, \ldots, i_{n} / O$ is correct for $M$ iff $i_{1} / o_{1}, \ldots, i_{n} / O$ is correct for $M$.

- If $i_{1} / o_{1}, \ldots, i_{n} / O$ is correct for $M$ and $O \subseteq O^{\prime}$ then $i_{1} / o_{1}, \ldots, i_{n} / O^{\prime}$ is correct for $M$.

- Let $I=i_{1} / o_{1}, \ldots, ? / o_{j}, \ldots, i_{n} / O$ be a correct invariant for $M$. Then, for any $I^{\prime}=i_{1} / o_{1}, \ldots, i / o_{j}, \ldots, i_{n} / O$, with $i \in \mathcal{I}$, such that $\exists s, s^{\prime} \in S: s \stackrel{I^{\prime}}{\longrightarrow} s^{\prime}$ we have that $I^{\prime}$ is correct for $M$.

- Let $i_{1} / o_{1}, \ldots, i_{j} / ?, \ldots, i_{n} / O$ be a correct invariant for $M$. Then, for any $I^{\prime}=i_{1} / o_{1}, \ldots, i_{j} / o, \ldots, i_{n} / O$, with $o \in \mathcal{O}$, such that $\exists s, s^{\prime} \in S: s \stackrel{I^{\prime}}{\longrightarrow} s^{\prime}$ we have that $I^{\prime}$ is correct for $M$. 
- Let $I$ be a correct invariant for $M$. If we consider the invariant $I^{\prime}$ where any occurrence of $*$ in $I$ is replaced by a sequence of symbols $i_{1} / o_{1}, \ldots, i_{j} / o_{j}$ such that $\exists s, s^{\prime}: s \stackrel{I^{\prime}}{\longrightarrow} s^{\prime}$ we have that $I^{\prime}$ is correct for $M$.

Let us note that the condition $s \stackrel{I^{\prime}}{\longrightarrow} s^{\prime}$ appearing in the last three cases indicates that there exists (at least) a pair of states such that they are connected by the sequence $I^{\prime}$. Moreover, the reverse implication of the last four results do not hold.

\subsection{Extending Invariants to Deal with EFSMs}

The extension of our framework to deal with EFSMs is far from trivial. In particular, we have the problem that the values of the variables cannot be, in general, observed. As we will comment in the conclusions of the paper, the work recently reported in $\left[\mathrm{LCH}^{+} 02\right]$ opens a new perspective for passive testing to cope with data values. Nevertheless, it is very easy to adapt our formalism to deal with invariants containing only constant data. Actually, this small inclusion is rather useful when dealing with real protocols as the WAP. For instance, we may use an invariant as

$$
\text { req_connect }(\text { Peter }) / ?, *, \text { req_disconnect }(\text { Peter }) /\{\text { disconnected }(\text { Peter })\}
$$

to check that the disconnection of the service performed by Peter is linked to a request of connection made by Peter himself.

\section{Applying Passive Testing with Invariants}

In this section we report our experiments with the WAP (Wireless Application Protocol) when using our notion of passive testing with invariants. We briefly present this protocol, we explain how our observation points are placed, and we discuss on the invariants that we have used.

\subsection{The Wireless Application Protocol}

The WAP is the standard protocol conceived to provide Internet content and advanced telephony services to wireless terminals. The Wireless Application Environment, in short WAE, is the main interface to the client device. It includes the content to be displayed (that is, a WML page). The WSP is a stateful binary protocol used in conjunction with WTP and WDP to provide session oriented services, or directly with WDP to provide connectionless service. It supports sessions initiation, suspension, and resumption. A session is initiated by a WAP client and is maintained until it is explicitly disconnected. WTP, is a confirmed transaction protocol, a light weighted version of TCP. There are three classes: A non-confirmed simple flow of information in one direction (class 0), a simple send-acknowledge exchange (class 1) and a class 2 for a three-way handshake. 


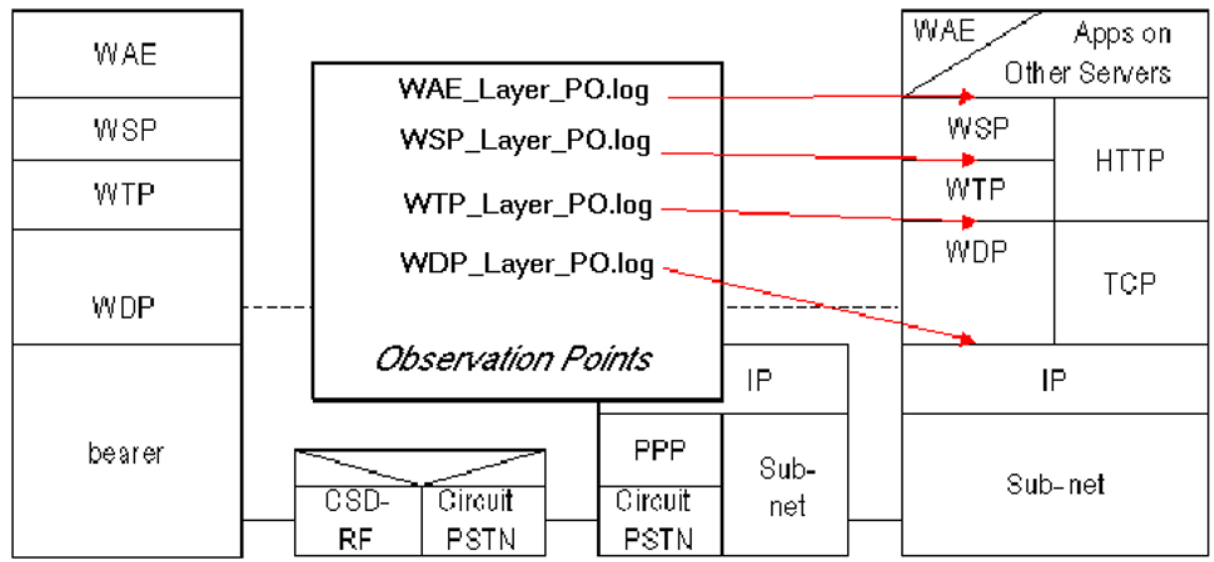

Mobile phone

IWF

Modem RAS

Gateway WAP

(Remote A ccess Server)

Fig. 4. A Passive Test Architecture for the WAP.

WTP also has an optional capability to segment and reassemble data. WDP, is a datagram oriented, network layer protocol. Its main purpose consists in making lower layers transparent to higher ones. It makes no delivery confirmation, packet retransmission or error correction. WTLS is a session oriented, secure protocol layer conceived after the Secure Session Layer (SSL) and Transaction Layer Security (TLS) protocols. This is optional and independent of the other parts. A schematic presentation of the protocol stacks is given in Figure 4.

We have developed an architecture capable of dealing with passive testing in a mobile phone environment (GSM-WAP). In order to do so, we deployed a platform that behaves as a normal WAP gateway ${ }^{3}$. In addition, we have included observation points, in short POs. These POs are placed in every layer of the WAP stack to show the flow of information in real time. So, whenever a communication between a mobile phone and a gateway exists, we have access to the involved messages and the information contained inside them. It is important to note that a layer is able to interpret only data belonging to the layer itself. This means that embedded data (i.e. from an upper layer) is not visible. The current state of every layer is also shown. These POs are entirely programmed in C. In order to have a closer control, an HTML interface with several PHP and CGI routines has also been developed.

\subsection{Experimental Results on the WAP}

For the sake of simplicity, in this paper we will only consider the PO that has been placed in the uppermost WAP layer, that is the session layer WSP. Thus, we can

3 That is, a network component that works as interface between the mobile phone side (wireless communication) and the Internet. The gateway we took is called Kannel. It is a free-software and it can be downloaded from http://www.kannel.org. 


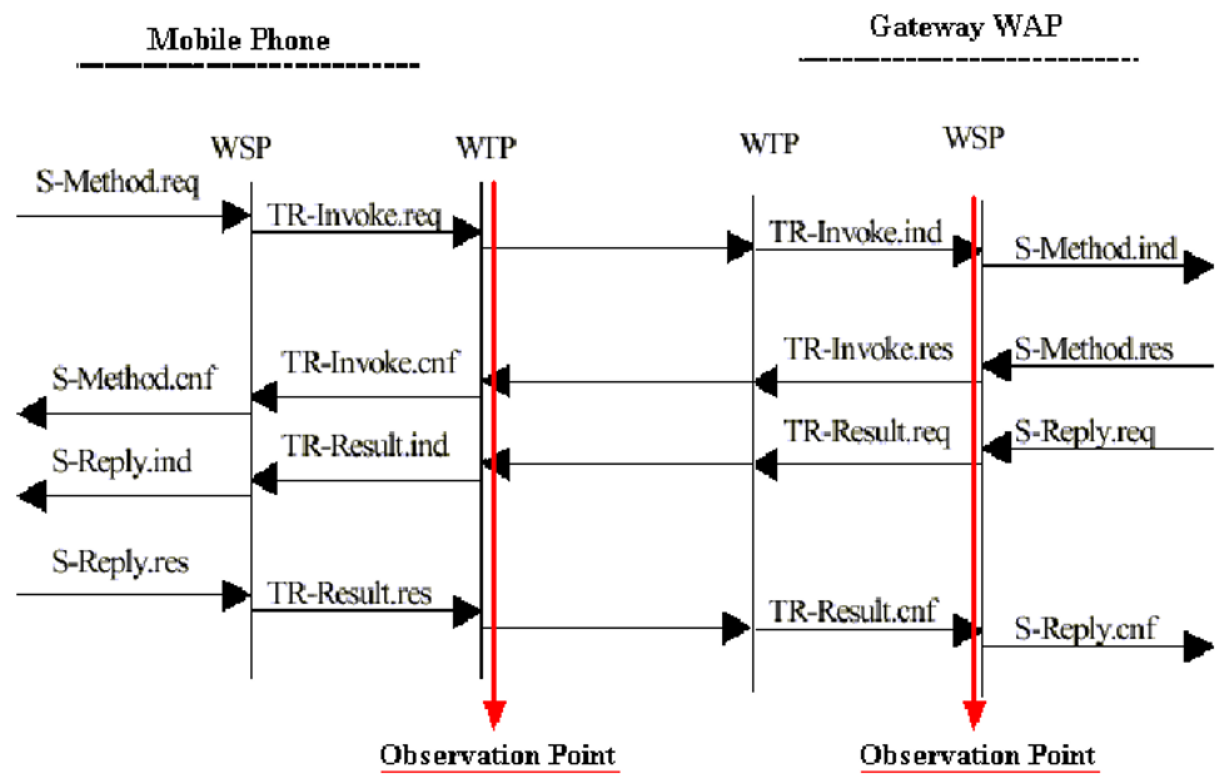

Fig. 5. Messages between layers and observation point.

observe those messages that are sent to or received from the lower layer WTP. The relation between these two layers, with respect to the transmitted messages, can be seen in Figure 5. The set of interesting events is given by: TR-Invoke, $T R$-Result, and TR-Abort. Each of these events may have one of the following attributes: req, ind, res, and cnf. Intuitively, a client $C$ sends and invoke message. This message is considered in the protocol as $T R$-Invoke.ind and it is received by the gateway $G$. Afterwards, an acknowledge TR-Invoke.res is sent. Then, $G$ tries to get the page requested by the user. Once $G$ gets the corresponding WML page, it sends a message to the client: TR-Result.req. The client receives this message as a TR-Result.ind event. Then, it sends an acknowledge, denoted by the event $T R$-Result.res. Finally, $G$ receives the event $T R$-Result.cnf denoting that the client received the requested information.

In order to test the protocol, we tried several properties extracted from the Wap Forum specification (see http://www.wapforum.org). The whole protocol was running autonomously and we were observing traces of 300 input/output pairs. Next we comment on the most relevant invariants that we were considering. First, we present a misleading example.

$$
\left.I_{1}=\text { TR-Invoke.req/?,*,TR-Result.res/\{TR-Result.cnf }\right\}
$$

This invariant is useful to check that whenever the client (mobile phone side) asks to download a WAP page, it is successfully received. More precisely, this is the way used by the WTP class 2 to acknowledge messages. Our experiments indicated that the traces were correct with respect to this invariant. However, we knew that this invariant was in fact not correct! Actually, an abort event can 
appear if the operation cannot be completed. For instance, this is the case if the requested web page is not available. So, we removed some of the requested web pages and we found that the new observed trace did not respect the invariant. In fact, the correct invariant is

$$
\left.I_{2}=\text { TR-Invoke.req/?,*,TR-Result.res/\{TR-Result.cnf,TR-Abort.ind }\right\}
$$

If we work within WTP class 0, no acknowledges are sent after a message is received. In this case, in order to check that the gateway sends the data to the cell phone we have to consider the event TR-Result.req. Thus, in WTP class 0 we have the invariant

$$
I_{3}=\text { TR-Invoke.req/?,*,TR-Result.req/ }\{\text { TR-Result.ind,TR-Abort.ind }\}
$$

Let us note that this invariant also holds in WTP class 2.

Our log files include a field called handle that uniquely identifies a given communication (between a cell phone and the gateway). Actually, any user of the protocol is uniquely identified. For instance, in GSM the identification is dynamically assigned each time the cell phone connects to the gateway: The modem assigns a dynamic IP. So, we may use our invariants to check some privacy aspects of the protocol. For example,

$$
I_{4}=\text { ?/TR-Invoke.ind(user1), *, TR-Result.res(user1)/O }
$$

where $O=\{T R$-Result.cnf(user1),TR-Abort.ind $\}$.

In the next table we summarize the main data related to the application of our invariants to the observed traces. We have normalized all the times with respect to the ones for $I_{1}$. In the first row we show the (normalized) times taken to check whether the invariants were correct. The second row indicates whether an error was found. Finally, the third row shows the (average and normalized) times obtained by matching the traces with the corresponding invariants. Note that the time associated with the first invariant is (much) smaller in this case because the process is stopped once a fault is detected

\begin{tabular}{|l|l|l|l|l|}
\hline Inv & $I_{1}$ & $I_{2}$ & $I_{3}$ & $I_{4}$ \\
\hline Time-Correct & 1 & 1.01 & 1.01 & 1.01 \\
\hline Error? & Yes & No & No & No \\
\hline Time-Trace & 1 & 8.72 & 8.46 & 9.22 \\
\hline
\end{tabular}

Finally, we would like to briefly comment on implementation details. We have developed a package where all the algorithms related to this paper are implemented. Besides, suitable interfaces between the corresponding modules have been implemented so that the process can be completely automatized. The code was written in $\mathrm{C}$ (on Linux) so that the whole test platform can be as portable as possible. As we have explained in the bulk of the paper, we have implemented a modification of the classical pattern matching algorithms. Specifically, the pattern matching algorithm for simple invariants is an adaptation of [KMP77] where pattern matching is performed from left to right. The algorithms for deciding 
whether invariants are correct with respect to a specification are completely original. The whole code for all of our algorithms and interfaces is around 1000 lines long.

\section{Conclusions and Future Work}

We have presented a new methodology for passive testing. Our approach to passive testing includes the definition of a new concept of invariant as well as the corresponding algorithms to deal with them. In addition, we give a new architecture to test properties on a given trace of the implementation. This methodology has been applied to the passive testing of the WAP protocol. Several properties have been tested and the results of experimentations are very promising.

Passive testing has very large domains of application. It can be used as a monitoring technique to detect and report errors (that is the use we consider in this paper). Other applications of passive testing are in network management to detect configuration problems, resource provisioning, etc. It can be also used to study the feasibility of new features: Classes of services, network security, congestion control, etc. We plan to continue our work on passive testing with invariants to the detection of errors in critical systems where active testing is not feasible. Another field of application that we are exploring is network security. We consider that our techniques are well adapted to detect anomalies, attacks and intrusions, but this point has to be further investigated. Finally, we would like to extend our invariants with capabilities so that not only the control part of protocols but also the data part can be taken into account. In this line, $\left[\mathrm{LCH}^{+} 02\right]$ represents a step forward in the passive testing methodology because data is formally considered. However, we still need to perform a more thorough study of this work in order to find out whether our invariants can be adapted to this new framework.

\section{References}

[AAD79] J.M. Ayache, P. Azema, and M. Diaz. Observer: A concept for on-line detection of control errors in concurrent systems. In 9th Symposium on Fault-Tolerant Computing, 1979.

[BM77] R.S. Boyer and J.S. Moore. A fast string searching algorithm. Communications of the ACM, 20:762-772, 1977.

[CGP01] A. Cavalli, C. Gervy, and S. Prokopenko. New approaches for passive testing using an extended finite state machine specification. In Concordia Prestigious Workshop on Communication Software Engineering, pages 225-250, 2001.

[KMP77] D.E. Knuth, J.H. Morris, and V.R. Pratt. Fast pattern matching in strings. SIAM Journal on Computing, 6(1):323-350, 1977.

[Lai02] R. Lai. A survey of communication protocol testing. Journal of Systems and Software, 62:21-46, 2002.

$\left[\mathrm{LCH}^{+}\right.$02] D. Lee, D. Chen, R. Hao, R. Miller, J. Wu, and X. Yin. A formal approach for passive testing of protocol data portions. In 10th IEEE Int. Conf. on Network Protocols, ICNP'02, pages 122-131. IEEE Computer Society Press, 2002. 
$\left[\mathrm{LNS}^{+}\right.$97] D. Lee, A.N. Netravali, K.K. Sabnani, B. Sugla, and A. John. Passive testing and applications to network management. In 5th IEEE Int. Conf. on Network Protocols, ICNP'97, pages 113-122. IEEE Computer Society Press, 1997.

[LY96] D. Lee and M. Yannakakis. Principles and methods of testing finite state machines: A survey. Proceedings of the IEEE, 84(8):1090-1123, 1996.

[MA01a] R.E. Miller and K.A. Arisha. Fault coverage in networks by passive testing. In International Conference on Internet Computing 2001, IC'2001, pages 413-419. CSREA Press, 2001.

[MA01b] R.E. Miller and K.A. Arisha. Fault identification in networks by passive testing. In 34th Simulation Symposium, SS'01, pages 277-284. IEEE Computer Society Press, 2001.

[Mil98] R.E. Miller. Passive testing of networks using a CFSM specification. In IEEE Int. Performance Computing and Communications Conference, pages 111-116. IEEE Computer Society Press, 1998.

[TC99] M. Tabourier and A. Cavalli. Passive testing and application to the GSMMAP protocol. Journal of Information and Software Technology, 41:813$821,1999$.

[TCI99] M. Tabourier, A. Cavalli, and M. Ionescu. A GSM-MAP protocol experiment using passive testing. In World Congress on Formal Methods in the Development of Computing Systems, FM'99, LNCS 1708, pages 915-934. Springer, 1999.

[WZY01] J. Wu, Y. Zhao, and X. Yin. From active to passive: Progress in testing of internet routing protocols. In FORTE 2001, pages 101-116. Kluwer Academic Publishers, 2001. 